\title{
ADICCIÓN ALIMENTARIA: ¿OTRO DETERMINANTE ASOCIADO A LA ADHESIÓN DEL TRATAMIENTO NUTRICIONAL?
}

\section{FOOD ADDICTION: ANOTHER DETERMINANT ASSOCIATED WITH THE ADHESION TO NUTRITIONAL TREATMENT?}

\section{DEPENDÊNCIA DE ALIMENTOS: OUTRO DETERMINANTE ASSOCIADO À ADESÃO DE TRATAMENTO NUTRICIONAL?}

\author{
Lucía Bentancor \\ Fondo Nacional de Recursos, Uruguay. Universidad Católica del Uruguay, Uruguay \\ luciabentancoruy@hotmail.com \\ ORCID: 0000-0003-3178-4533 \\ Soledad Calvo \\ Fondo Nacional de Recursos, Uruguay. Universidad Católica del Uruguay, Uruguay \\ ORCID: 0000-0001-5894-7369
}

\section{Gustavo Saona}

Fondo Nacional de Recursos, Uruguay

ORCID: 0000-0003-1329-7475

DOI: $10.22235 /$ ech.v8i2.1848

Recibido: 14/02/2019

Aceptado:04/07/2019

\section{RESUMEN:}

Los Programas de Prevención Secundaria Cardiovascular se han desarrollado para mejorar el proceso de atención de pacientes con cardiopatía isquémica y/o con alto riesgo cardiovascular. Sin embargo, la adherencia terapéutica en algunos aspectos es limitada, por lo que conocer la prevalencia de adicción alimentaria en esta población podría ser de utilidad para redireccionar la intervención nutricional. El objetivo de este trabajo es estudiar la prevalencia de adicción alimentaria de los pacientes que asisten al Programa de Prevención Secundaria Cardiovascular del Fondo Nacional de Recursos en el período octubre a diciembre 2016 en el departamento de Montevideo, Uruguay. Se trata de un estudio descriptivo, observacional y de corte transversal. Se estudiaron 153 pacientes a los que se les aplicó el cuestionario estructurado YFAS de Gearhardt y valoración del estado nutricional a través del IMC extraído del Sistema Informático "María" del FNR. El 63\% de la población fue del sexo masculino, 8 de cada 10 de los encuestados fueron adultos mayores ( $\geq 60$ años), y predominó la malnutrición por exceso en el $70 \%$ de los encuestados, encontrándose obesidad en 4 de cada 10. La prevalencia de adicción alimentaria en el total de los encuestados fue del 5,9\% (IC 95\% = 2,7-10,9), siendo más prevalente en el rango etario de menores de 60 años. Dentro de la categoría de obesidad la prevalencia de adicción alimentaria fue del 13,8\%, seguida por el sobrepeso. Se observa en un $10 \%$ de la población la presencia de tres o más síntomas para adicción alimentaria sin tener aún la significación clínica que determine la presencia de la patología, predominando ésto en la población del sexo masculino y en los menores de 60 años. Se concluye que casi el 6\% de la población presentó adicción alimentaria y que un $10 \%$ tuvo tres o más síntomas propios de la patología. Incluir el test en la consulta de los pacientes pertenecientes al PPSCV permitiría derivar al equipo de psicología de la institución los pacientes que así lo requieran.

Palabras Clave: Adicción a la Comida, Evaluación Nutricional, Encuestas Nutricionales. 


\section{SUMMARY:}

Cardiovascular Secondary Prevention Programs have been developed to improve the care process for patients with ischemic heart disease and/or high cardiovascular risk. However, the therapeutic adherence in some aspects is limited, so knowing the prevalence of food addiction in this population could be useful to redirect the nutritional intervention. The objective of this work is to study the prevalence of food addiction in patients attending the Cardiovascular Secondary Prevention Program (CSPP) of the National Resources Fund (NRF) from October to December 2016 in Montevideo, Uruguay. It is a descriptive, observational, cross-sectional study with 153 patients. The structured YFAS questionnaire of Gearhardt was applied and the nutritional status was assessed through the BMI data extracted from the "Maria" IT System of the NRF. $63 \%$ of the population was male, 8 out of 10 of the respondents were over 60 years of age, and overnutrition predominated in $70 \%$ of the respondents, with obesity present in 4 out of 10 . The prevalence of food addiction in the total of the respondents was 5.9\% (95\% CI $=2.7-10.9)$, being more prevalent in the age range of those under 60 . Within the category of obesity, the prevalence of food addiction was $13.8 \%$, followed by being overweight. The presence of three or more symptoms for food addiction was observed in $10 \%$ of the population, without still having the clinical significance that determines the presence of the pathology, predominating in the male population and in those under 60 . It is concluded that almost $6 \%$ of the population had food addiction and $10 \%$ had three or more symptoms of the pathology. Including the test in the consultation of patients belonging to the Program would allow referral of the patients requiring it to the psychology team of the institution.

Key words: Food Addiction, Nutritional Evaluation, Nutrition Surveys.

\section{RESUMO:}

Programas secundários de prevenção cardiovascular foram desenvolvidos para melhorar o processo de atendimento de pacientes com cardiopatia isquêmica e / ou com alto risco cardiovascular. No entanto, a adesão terapêutica em alguns aspectos é limitada, portanto, conhecer a prevalência de dependência alimentar nessa população pode ser útil para redirecionar a intervenção nutricional. O objetivo deste trabalho é estudar a prevalência de dependência alimentar de pacientes do Programa de Prevenção Secundária Cardiovascular do Fundo Nacional de Recursos no período de outubro a dezembro de 2016 no departamento de Montevidéu, Uruguai. Trata-se de um estudo descritivo, observacional e transversal. Foram estudados 153 pacientes aos quais foi aplicado o questionário estruturado YFAS de Gearhardt e a avaliação do estado nutricional por meio do IMC extraído do María Informatics System do FNR. 63\% da população era do sexo masculino, 8 em cada 10 entrevistados eram adultos mais velhos $(\geq 60$ anos) e a supernutrição prevaleceu em $70 \%$ dos entrevistados, com obesidade em 4 em cada 10. a prevalência de dependência alimentar no total dos entrevistados foi de 5,9\% (IC95\% = 2,7-10,9), sendo mais prevalente na faixa etária de crianças menores de 60 anos. Na categoria obesidade, a prevalência de dependência alimentar foi de 13,8\%, seguida pelo excesso de peso. Observa-se em $10 \%$ da população a presença de 3 ou mais sintomas de dependência alimentar sem ainda ter o significado clínico que determina a presença da patologia, predominantemente na população masculina e naqueles com menos de 60 anos. Conclui-se que quase $6 \%$ da população apresentava dependência alimentar e 10\% apresentavam 3 ou mais sintomas da patologia. A inclusão do teste na consulta de pacientes pertencentes ao PPSCV permitiria que os pacientes que precisassem dele se referirem à equipe de psicologia da instituição.

Palavras-chave: Dependência de Alimentos, Avaliação Nutricional, Inquéritos Nutricionais. 


\section{INTRODUCCIÓN}

La obesidad es una enfermedad multifactorial: abarca tanto los factores genéticos, el sexo, la edad, como la educación, lo económico, social y cultural de cada individuo. Asimismo, hay otro factor de gran relevancia, muchas veces ignorado: los aspectos psicológicos de la enfermedad. Un punto importante dentro de este factor es la percepción de la imagen corporal (IC). En un estudio realizado a pacientes que asisten al Programa de Prevención Secundaria Cardiovascular del Fondo Nacional de Recursos (PPSCV del FNR) se observa que la mayoría de los pacientes presentan una subestimación severa de su IC: se percibían con un peso más bajo del que tenían. Los obesos se percibieron con sobrepeso, y estos últimos con un peso normal (1). Si la persona no se percibe con un problema de malnutrición por exceso no cambiará aspectos de sus hábitos para poder modificar su situación y de esa forma reducir los riesgos de desarrollar enfermedades crónicas.

Dentro del factor psicológico se encuentra la Adicción Alimentaria (AA) como un factor clave. La palabra adicción proviene de latín addictio: deudor, por falta de pago, que era entregado como esclavo a su acreedor (2). Según la Organización Mundial de la Salud (OMS), una adicción es una enfermedad física y psicoemocional; es una dependencia o necesidad hacia una sustancia, actividad o relación (codependencia) (2). El consumo repetido de alimentos ricos en azúcares y grasas da lugar a una regulación disminuida de los receptores de dopamina (DA) y una disminución de la sensibilidad del circuito meso simbólico de recompensa; estos cambios ocurren en respuesta al abuso de sustancias en conductas adictivas como el alcohol, marihuana, cocaína y heroína. La disminución de la actividad de DA en el área tegmental ventral (ATV) conduce un aumento importante en el consumo de alimentos (3). En 1956 Randolph describió la existencia de individuos que presentaban síntomas relacionados con determinados alimentos, con un patrón similar a lo observado en la adicción (4). La repetida exposición a ciertos alimentos, particularmente alimentos de elevada densidad energética, en individuos vulnerables puede desencadenar un consumo compulsivo y un bajo control sobre la ingesta; de esta manera surge el término de adicción alimentaria (AA) (4).

Existen varias investigaciones y estudios a nivel mundial, donde evalúan la AA en determinadas poblaciones. De esos estudios se destaca que la prevalencia de AA en poblaciones de estudiantes y comunidad en general oscila entre el 5-10\%, incrementándose en poblaciones obesas hasta un 15-25\%; este número aumenta aún más en poblaciones con obesidad mórbida (5). A nivel local, en Latinoamérica más precisamente, existe un estudio realizado en México y otro en Chile. El primero es una validación del Yale Food Adiction Scale (YFAS) y su traducción al español. El segundo evalúa la presencia de AA en estudiantes chilenos y lo relaciona directamente con su 
estado nutricional. En este estudio se observa que esta condición es más prevalente en mujeres que en hombres y que está relacionada con la obesidad $(4,6)$.

Los Programas de Prevención Secundaria Cardiovascular (PPSCV) se han desarrollado para mejorar el proceso de atención de pacientes con cardiopatía isquémica y/o con alto riesgo cardiovascular. El objetivo del Programa es optimizar el tratamiento de los pacientes con enfermedad coronaria, promover estilos de vida saludables, controlar los factores de riesgo cardiovascular y facilitar el acceso a la medicación cardioprotectora para mejorar la sobrevida, la calidad de vida y disminuir la necesidad de nuevos procedimientos de revascularización(7).

El presente estudio busca estudiar la prevalencia de AA en los pacientes asistentes al PPSCV para optimizar las herramientas ya usadas para su tratamiento y generar la posibilidad de crear nuevas, favoreciendo de esta forma la atención integral del paciente en la consulta cardiovascular. El objetivo es I) estudiar la prevalencia de adicción alimentaria de los pacientes que asisten al Programa de Prevención Secundaria Cardiovascular del Fondo Nacional de Recursos en el período Octubre-Diciembre 2016 en el departamento de Montevideo; II) Caracterizar a la población de estudio según edad, sexo y estado nutricional; y, III) Conocer la prevalencia de adicción alimentaria en los pacientes.

\section{METODOLOGÍA}

Se realizó un estudio descriptivo, observacional y de corte transversal. La muestra fue no probabilística por conveniencia de 153 pacientes. Se incluyeron a los pacientes asistentes al PPSCV que hayan concurrido a consulta entre octubre y diciembre 2016, que se contaran con todos los datos necesarios para evaluar las variables propuestas y que estuvieran en condiciones de responder a las preguntas. Para la caracterización de la población de estudio se utilizaron los datos que se encuentran en el Sistema informático "María"del FNR.

Las variables utilizadas para caracterizar la población en estudio fueron la edad ( $<60$ años ó $\geq 60$ años), sexo (femenino o masculino), y estado nutricional. Para este último se diferenciaron las categorías de acuerdo a la edad del paciente ( $<60$ años ó $\geq 60$ años) ya que para evaluar adultos mayores los criterios de IMC utilizados son diferentes: $\leq 60$ años= bajo peso (IMC $<18,5)$, normopeso (IMC 18,5-24,9), sobrepeso (IMC $\geq 25-29,9$ ), obesidad (IMC $\geq 30$ ). Mientras que los adultos mayores ( $\geq 60$ años) bajo peso (IMC $<23$ ) normopeso (IMC 23-27,9), Sobrepeso (IMC $\geq 28-31,9$ ), Obesidad ( $\geq 32$ ) - OPS 2002. Para medir la AA se utilizó la escala YFAS, la cual se aplicó de forma telefónica a casi la totalidad de la población y personal ( $3 \%$ de la población de estudio) a la muestra seleccionada.

Gearhardt y otros desarrollaron un instrumento basado en el DSM-IV-TR para determinar aquellos individuos con alto riesgo de adicción a la comida, independientemente de su peso (4). Consiste en un cuestionario de 25 preguntas en formato dicotómico y escala tipo Likert, donde se les solicita a los sujetos que se refieran a sus comportamientos relativos 
a la alimentación en los últimos doce meses. Este cuestionario permite diferenciar individuos que presentan AA de los que no, como también observar individuos que presentan los síntomas. El uso de este instrumento muestra que mayores puntajes de adicción a la comida se correlacionan con una mayor activación de regiones que están implicadas en la dependencia de sustancias, en respuesta a determinados alimentos. Se utilizó la traducción al español de la escala realizada por Valdés y cols (6). Para el análisis de los resultados se utilizó una matriz diseñada en Excel extraída de la página del Laboratorio de Ciencia y Tratamiento de Alimentos y Adicciones.

Se realizó una prueba piloto de aplicación de la encuesta a un grupo de pacientes que se asisten en el Programa para la Atención a la Obesidad con el fin de constatar el entendimiento de la misma por parte de los mismos. Si bien la muestra fue pequeña (10 participantes) de la misma se desprende que el 10\% de los encuestados presentó adicción a la comida entendida por la presencia de tres o más síntomas y presencia de significación clínica. De los restantes se destaca que, si bien no presentaron adicción por no cumplir con los criterios establecidos para la misma, es de interés que el $44 \%$ presenta tres o más síntomas de adicción, dato no menor a tener en cuenta en la atención que se les brinda puesto que puede interferir con el éxito del tratamiento.

\section{RESULTADOS}

La población de estudio consistió en un total de 153 pacientes, el 63\% de sexo masculino. Con respecto al rango etáreo de predominio, el $81 \%$ de la población fue $\geq 60$ años, clasificándose como adultos mayores. Analizando el estado nutricional de estos pacientes se desprende que el $70 \%$ de la población presentó malnutrición por exceso, $32 \%$ sobrepeso y el $38 \%$ obesidad.

Tabla 1: Distribución de los encuestados según estado nutricional y edad

\begin{tabular}{|l|c|c|c|c|}
\hline \multicolumn{1}{|c|}{$\begin{array}{c}\text { ESTADO } \\
\text { NUTRICIONAL }\end{array}$} & \multicolumn{2}{c|}{ EDAD } & \multicolumn{2}{c|}{ TOTAL } \\
\hline & $<\mathbf{6 0}$ años & $\geq \mathbf{6 0}$ años & FA & FR \% \\
\hline Obesidad & 20 & 38 & 58 & 38 \\
\hline Sobrepeso & 8 & 41 & 49 & 32 \\
\hline Normal & 1 & 38 & 39 & 25 \\
\hline Bajo peso & 0 & 7 & 7 & 5 \\
\hline TOTAL & 29 & 124 & 153 & $100 \%$ \\
\hline
\end{tabular}

Fuente: Fondo Nacional de Recursos, Octubre-Diciembre (2016) 
Tabla 2: Distribución de los encuestados según sexo y estado nutricional

\begin{tabular}{|l|l|l|l|l|l|l|}
\hline $\begin{array}{l}\text { ESTADO } \\
\text { NUTRICIONAL }\end{array}$ & \multicolumn{2}{|l|}{ Masculino } & \multicolumn{2}{l|}{ Femenino } & \multicolumn{2}{l|}{ TOTAL } \\
\hline & FA & FR \% & FA & FR \% & FA & FR \% \\
\hline Obesidad & 40 & 26 & 18 & 12 & 58 & 38 \\
\hline Sobrepeso & 31 & 20 & 18 & 12 & 49 & 32 \\
\hline Normal & 22 & 14 & 17 & 11 & 39 & 25 \\
\hline Bajo peso & 4 & 3 & 3 & 2 & 7 & 5 \\
\hline TOTAL & 97 & 63 & 56 & 37 & 153 & $100 \%$ \\
\hline
\end{tabular}

Fuente: Fondo Nacional de Recursos, Octubre-Diciembre (2016)

Gráfico 1: Distribución de los encuestados según sexo y estado nutricional

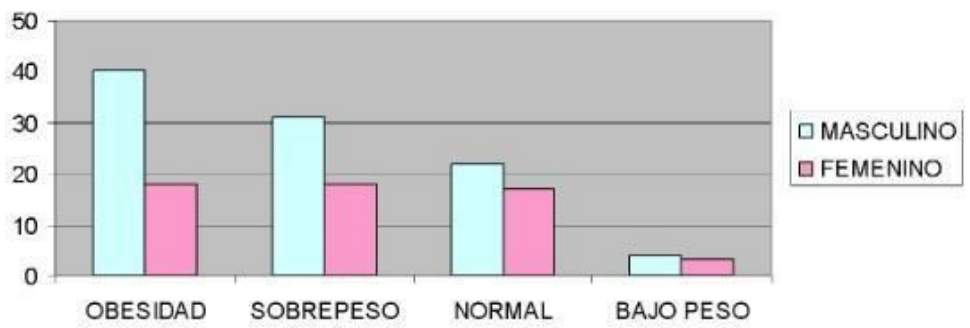

Fuente: Fondo Nacional de Recursos, Octubre-Diciembre (2016)

Si se toma en cuenta además el estado nutricional y el sexo, se observa que la mitad de los encuestados que tienen sobrepeso u obesidad son hombres (Tabla 2). La mayor parte de las encuestas fueron en forma telefónica (97\%). Con respecto a la prevalencia de AA en esta población se observa que el 5,9\% (IC 95\% = 2,7-10,9) de la muestra cumple con los criterios YFAS, esto quiere decir que presenta tres o más síntomas de la patología y que además asocia significación clínica (preguntas 15 y 16 de la escala mayor a 1 (Gráfico $\mathrm{N}^{\circ}$ 2).

Gráfico 2: Distribución de los encuestados según presencia de adicción alimentaria

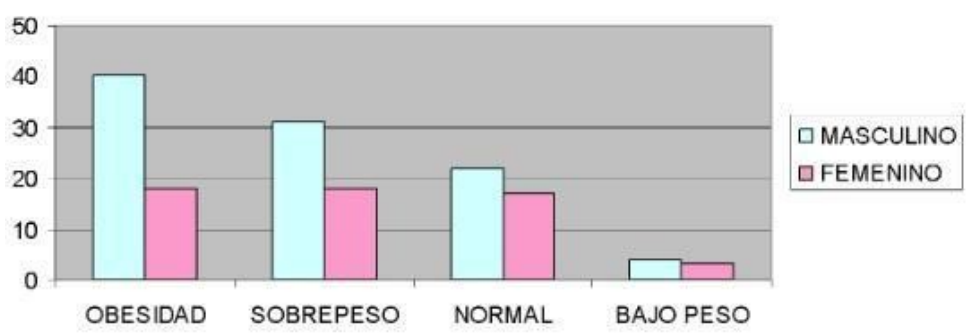

Fuente: Fondo Nacional de Recursos, Octubre-Diciembre (2016) 
Tabla 3: Distribución de los pacientes según sexo y adicción alimentaria

\begin{tabular}{|l|l|l|l|}
\hline $\begin{array}{l}\text { Adicción } \\
\text { alimentaria } \\
\text { Sexo }\end{array}$ & FR \% & FA & TOTAL \\
\hline Masculino & $6,2 \%(2,3-13)$ & 6 & 97 \\
\hline Femenino & $5,6 \%(1,1-14,9)$ & 3 & 56 \\
\hline
\end{tabular}

Fuente: Fondo Nacional de Recursos, Octubre-Diciembre (2016)

No se observó relación entre adicción y sexo, ya que se encontraron valores similares de prevalencia de adicción tanto en el grupo de mujeres como en el de hombres (Tabla N³).

Tabla 4: Distribución de los pacientes según edad y adicción alimentaria

\begin{tabular}{|l|l|l|l|}
\hline $\begin{array}{l}\text { Adicción alimentaria } \\
\text { Edad }\end{array}$ & FR \% & FA & TOTAL \\
\hline$<60$ años & $20,7 \%(8-39,7)$ & 6 & 29 \\
\hline$\geq 60$ años & $2,4 \%(0,5-6,9)$ & 3 & 124 \\
\hline
\end{tabular}

Fuente: Fondo Nacional de Recursos, Octubre-Diciembre (2016)

Al observar la edad, se destaca una mayor prevalencia de AA en el rango etáreo de menores de 60 años (Tabla 4).

Tabla 5: Distribución de los pacientes según estado nutricional y adicción alimentaria

\begin{tabular}{|l|l|l|l|}
\hline $\begin{array}{l}\text { Adicción alimentaria } \\
\text { Estado nutricional }\end{array}$ & FR \% & FA & TOTAL \\
\hline Obesidad & $13,8 \%(6,1-25,4)$ & 8 & 58 \\
\hline Sobrepeso & $2 \%(0-10,9)$ & 1 & 49 \\
\hline Normal & $0 \%(0,7-4)$ & 0 & 39 \\
\hline Bajo peso & $0 \%(0-34,9)$ & 0 & 7 \\
\hline
\end{tabular}

Fuente: Fondo Nacional de Recursos, Octubre-Diciembre (2016)

Teniendo en cuenta el estado nutricional de los pacientes de la muestra, se destaca que dentro de la categoría de obesidad la prevalencia de AA es 13,8\% seguida por el sobrepeso (Tabla 5). 
Tabla 6: Distribución de los pacientes que no tienen adicción alimentaria según cantidad de síntomas que presentan

\begin{tabular}{|l|l|l|}
\hline $\begin{array}{l}\text { Síntomas de Adicción } \\
\text { Alimentaria }\end{array}$ & FA & FR \% \\
\hline$<3$ síntomas & 130 & $90 \%$ \\
\hline$\geq 3$ síntomas & 14 & $10 \%$ \\
\hline TOTAL & 144 & $100 \%$ \\
\hline
\end{tabular}

Fuente: Fondo Nacional de Recursos, Octubre-Diciembre (2016)

Tabla 7: Distribución de los pacientes con más de 3 síntomas según sexo

\begin{tabular}{|l|l|l|l|}
\hline Síntomas de AA & FA & FR \% & TOTAL \\
Sexo & & & \\
\hline Masculino & 12 & $12,4 \%(6,6-20,6)$ & 97 \\
\hline Femenino & 2 & $3,6 \%(0-12,3)$ & 56 \\
\hline
\end{tabular}

Fuente: Fondo Nacional de Recursos, Octubre-Diciembre (2016)

Tabla 8: Distribución de los pacientes con más de 3 síntomas según edad

\begin{tabular}{|l|l|l|l|}
\hline Síntomas de AA & FR \% & FA & TOTAL \\
Edad & & & \\
\hline$<60$ años & $10 \%((2,2-27,4)$ & 3 & 29 \\
\hline$\geq 60$ años & $8,9 \%(4,5-15,3)$ & 11 & 124 \\
\hline
\end{tabular}

Fuente: Fondo Nacional de Recursos, Octubre-Diciembre (2016)

Tabla 9: Distribución de los pacientes con más de 3 síntomas según estado nutricional

\begin{tabular}{|l|l|l|l|}
\hline Síntomas de AA & FR \% & FA & TOTAL \\
\hline Obstado nutricional & & & \\
\hline Sobrepeso & $10 \%(3,9-21,2)$ & 6 & 58 \\
\hline Normal & $4 \%(0,5-14)$ & 2 & 49 \\
\hline Bajo peso & $10 \%(2,9-24,2)$ & 4 & 39 \\
\hline
\end{tabular}

Fuente: Fondo Nacional de Recursos, Octubre-Diciembre (2016) 
Cabe destacar que hay un $10 \%$ de la población que presenta tres o más síntomas para AA sin tener aún la significación clínica que determine la presencia de la patología, predominando esto en la población del sexo masculino $(12,4 \%)$ y en los menores de 60 años $(10 \%)$. Tomando en cuenta la asociación de presencia de sintomatología de AA y estado nutricional, se observa que hay un $10 \%$ de los obesos que tienen tres o más síntomas para AA y un 4\% de los pacientes con sobrepeso, mientras que en la población con estado nutricional normal hay un $10 \%$ que tienen estos síntomas. Estos valores fueron superados por los pacientes con bajo peso, pero estos resultados están afectados por el bajo tamaño de la muestra (IC95\% = 3,7-71). Un punto que es de suma importancia a resaltar es que todos los pacientes que integraron la muestra presentan por lo menos un síntoma de AA.

Gráfico 3: Distribución de los pacientes según tipo de síntoma y estado nutricional

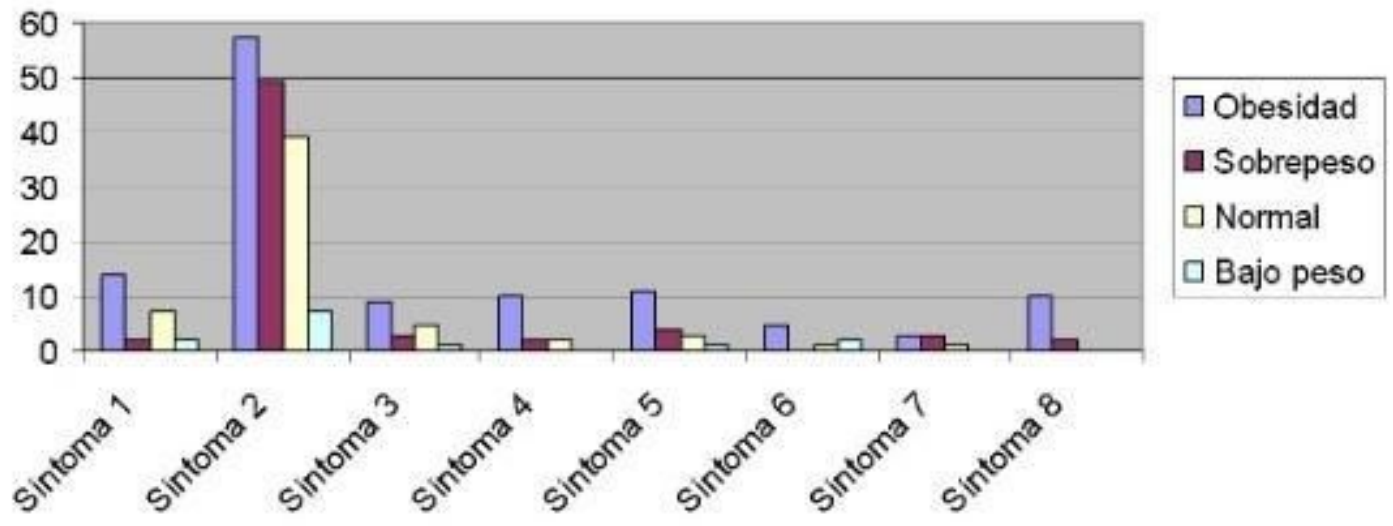

Fuente: Fondo Nacional de Recursos, Octubre-Diciembre (2016)

A través del gráfico anterior se puede observar qué síntomas de adicción alimentaria predominaron por estado nutricional.

Síntomas:

1- Sustancia tomada en mayor cantidad y por un período más largo de lo previsto.

2- Deseo persistente o repetidos intentos fallidos de dejar de comer.

3- Mucho tiempo/ actividad para obtener, usar, recuperar.

4- Importantes actividades sociales, ocupacionales o recreativas dadas o reducidas.

5- El uso continúa a pesar del conocimiento de las consecuencias adversas (por ejemplo, el incumplimiento de la obligación del papel, el uso cuando es físicamente peligroso). 6- Tolerancia (aumento marcado en la cantidad, marcada disminución en el efecto). 7- Síntomas de abstinencia característicos, sustancia tomada para aliviar la retirada. 8- El uso causa deterioro o angustia clínicamente significativa.

En obesidad los síntomas 2, 1 y 5 son los que destacan. Un dato no menor, es que para todos los pacientes sea cual sea su estado nutricional, el síntoma 2 es el de mayor prevalencia, y tiene que ver con los deseos e intentos por dejar o reducir el consumo de determinados alimentos (Gráfico 3). 


\section{DISCUSIÓN}

En Uruguay casi el $65 \%$ de la población presenta sobrepeso u obesidad. Los datos arrojados en este estudio no se apartan de la realidad, excediendo el porcentaje nacional. Las características tanto de edad, sexo y estado nutricional se correlacionan directamente con el estudio realizado en el FNR (2004-2007), donde la mayoría de la población ingresada fue del sexo masculino, comprendidos entre 30 y 70 años, predominando la obesidad.

La malnutrición por exceso, más específicamente la obesidad, resulta de una transformación en la forma de alimentación y de la actividad física, comprendiendo en sí mismas la llamada "transición nutricional" (8). Este aumento desmedido del porcentaje de obesos a nivel mundial obligó a profundizar su estudio y buscar nuevas causas que fueran el puntapié inicial para el desarrollo de políticas públicas que atacaran el problema, tendiendo a disminuirlo.

Si bien se buscaron y se siguen buscando nuevas causas, en los últimos años se ha puesto mucho énfasis en trabajar los aspectos psicológicos que influyen en el desarrollo y mantenimiento de la obesidad. En esta línea, se estudió la percepción de la imagen corporal encontrándose en varios estudios a nivel mundial una distorsión en la percepción de la misma, dónde la mayoría de las personas que integraron los estudios subestimaron su peso $(9,10)$. Un estudio similar se realizó en pacientes asistentes al PPSCV del FNR habiéndose medido a través de las siluetas de Stunkard, encontrándose de la misma forma que a nivel mundial con un porcentaje muy elevado de pacientes que subestimaron su peso corporal (11).

La normalización y aceptación de la obesidad a nivel social y la persistencia de valores de paraclínica alterados en estos pacientes a pesar de todos los recursos tanto materiales como humanos que se utilizan en su atención, hizo necesaria la búsqueda de otras causas que pudieran estar interfiriendo con la adherencia al tratamiento. La prevalencia de AA fue de casi un $6 \%$, siendo este valor similar al encontrado en poblaciones de estudiantes y comunidad en general (5-10\%). En 2015, en Chile, se aplicó el cuestionario YFAS en una población de universitarios arrojando como resultados que la AA era más prevalente en mujeres y que estaba relacionada con la obesidad. En una muestra de adultos obesos se encontró una prevalencia de AA del $25 \%$. En obesos que desean perder peso las tasas aumentan aún más, cumpliendo los criterios de AA en un $41,7 \%$ y $53,7 \%$ en pacientes que solicitan cirugía bariátrica. En el presente estudio también se puede asociar la AA con el exceso de peso ya que de los obesos un 13,8\% presentó AA, mientras que quienes tenían sobrepeso alcanzó un $2 \%$. No es posible relacionar los resultados de la investigación citada con respecto a la prevalencia según sexo, ya que en esta investigación la prevalencia de AA fue similar en los dos sexos.

En un estudio de evaluación de la escala de adicción a los alimentos de Yale se correlacionó negativamente la presencia de sintomatología para desarrollar AA con la pérdida de peso, lo que sugiere que esta sintomatología podría afectar el éxito del tratamiento en personas obesas (5). En el presente estudio se pudo observar que toda la población presentó al menos un síntoma para AA, y un $10 \%$ tuvo tres o más síntomas, siendo más prevalente dentro del sexo masculino y en menores de 60 años. Si se 
considera además el estado nutricional donde prevalecen estos síntomas, se observa que hay un $10 \%$ en obesidad y un $4 \%$ en sobrepeso. Es importante destacar que en la población con estado nutricional normal hay un 10\% que tienen estos síntomas. Dichos valores fueron superados por los pacientes con bajo peso, pero estos resultados están afectados por el bajo tamaño de muestra (IC 95\%=3,7-71), recomendándose para un estudio futuro el aumento del tamaño de la misma y la posibilidad de realizar una investigación similar solamente con pacientes con bajo peso.

Uno de los síntomas claves de esta patología es el "craving”, el deseo o ansia apremiante, urgente e irreprimible, lo que resulta en la mayoría de los casos en una pérdida de control. Esto contribuye en el desarrollo, mantenimiento y recaída de una conducta adictiva. Al analizar los síntomas relacionados al estado nutricional se observa que sea cual sea el mismo, el deseo persistente o repetido por dejar de consumir determinados alimentos está presente. En obesidad el síntoma que alude al consumo en mayor cantidad y por períodos más largos de lo previsto es el que predomina, y casi en la misma proporción declararon el uso continuo a pesar del conocimiento de las consecuencias adversas que produce. En un estudio realizado a personas obesas en Estados Unidos y Australia en 2014 se observó que el $86 \%$ de la población tuvo deseo persistente o intentos infructuosos de reducir ciertos alimentos, y el 29\% informaron continuar consumiendo ciertos alimentos a pesar de los problemas psicológicos o físicos derivados de dicho uso de alimentos (12). Es en estos aspectos (síntomas) en los que se puede intervenir para prevenir el desarrollo de la AA propiamente dicha en los pacientes que aún no la presentan. Es de gran importancia conocer los tratamientos que actualmente se están utilizando para seleccionar aquellos que sean los más adecuados y aplicables en este grupo de pacientes.

Los tratamientos de primera elección para abordar las adicciones, y en particular para el "craving", se basan en la utilización de fármacos y/o tratamientos psicoterapéuticos. Aquí se observa un punto en el cual se pueden realizar intervenciones para prevenir el desarrollo de la patología en los pacientes asistentes al PPSCV. Dentro de los tratamientos psicológicos y/o conductuales se encuentra la Terapia Cognitivo Conductual (TCC) que ha mostrado resultados satisfactorios en la reducción de síntomas de AA y psicopatología alimentaria (13). Ésta permite al paciente observar su conducta, pensamientos y emociones y su relación con sus hábitos alimentarios inadecuados para buscar alternativas más apropiadas. Las intervenciones nutricionales permiten a los pacientes mantener hábitos alimentarios saludables a través de la psicoeducación y el establecimiento de patrones alimentarios regulares como el manejo de situaciones de riesgo.

Por otra parte, la Terapia Interpersonal se ha utilizado con buenos resultados en pacientes con sobrepeso y obesidad que refieren pérdida de control de ingesta. Se centra en los problemas interpersonales, mejora el estado de ánimo y aumenta los sentimientos de autoeficacia de los pacientes. La Terapia Dialéctica Conductual (TDC) fue adaptada para su aplicación en trastornos alimentarios, y se centra en la regulación emocional. También se han comenzado a usar las nuevas tecnologías, como la realidad virtual, que simula un entorno tridimensional generado por ordenador y permite al usuario interactuar en tiempo real con una interfaz que facilita un sentimiento de presencia en dicho entorno y el juicio de realidad de su experiencia. 
Los tratamientos farmacológicos son similares a los aplicados en pacientes con dependencia a sustancias. En primer lugar, los antagonistas no selectivos de los receptores opioides, como la naltrexona, podrían disminuir el deseo y consumo de alimentos ricos en azúcar y grasas en personas en normopeso y en pacientes obesos con diagnóstico de bulimia nerviosa $u$ otro trastorno alimentario (13). Por otra parte, los fármacos inhibidores selectivos de la recaptación de serotonina disminuyen los episodios de atracones. Los tratamientos basados en el glutamato, tanto el acamprosato como el topiramato han mostrado resultados positivos, disminuyendo el "craving" por la comida y controlando el aumento de peso en pacientes con dependencia al alcohol. Se sugiere que las técnicas de neuromodulación para tratar la adicción a la comida y más concretamente el "craving" podrían ser de utilidad (13).

\section{CONCLUSIÓN}

El PPSCV del FNR atiende al paciente de forma integral, tratando de abarcar todos los aspectos que hacen a su patología con el fin de contribuir a una mejor calidad de vida. En los últimos datos obtenidos de un estudio realizado a esta población se observó que aproximadamente 3 de cada 10 pacientes presentan obesidad y no cumplen con la recomendación de consumo de frutas, verduras, pescado o cortes de carne magra. A su vez, el consumo de grasa es inadecuado (presencia de fritos, manteca, margarina). A pesar de los esfuerzos realizados, existe un número importante de pacientes que no adhiere en algún aspecto del tratamiento. El presente estudio buscó otro factor que pudiera estar influyendo de forma negativa en la adhesión de estos pacientes, y se observó que casi el 6\% de la población presentó AA. Un dato a destacar es que existe un $10 \%$ que si bien no presentan AA tienen tres o más síntomas propios de la patología, lo que podría desencadenar en una adicción si no se trata a tiempo. Es de destacar que todos los integrantes de la muestra tienen al menos uno de los síntomas. También se observó que dentro de los pacientes que presentan obesidad, 1 de cada 10 tiene AA. Siendo un tema que hace pocos años está tomando relevancia y teniendo acceso al test que nos permite evaluar la presencia de esta patología, sería conveniente que se considerara una herramienta más en la atención de los pacientes pertenecientes al PPSCV, pudiendo en los casos correspondientes derivarse al equipo de psicología de la institución.

\section{REFERENCIAS BIBLIOGRÁFICAS}

1. Riccetto P, Calvo S, Gambogi R, Nigro S, Sánchez F. Percepción de imagen corporal, factor clave en el cuidado del estado nutricional. Fondo Nacional de Recursos. Montevideo, Uruguay; 2015.

2. Facchini M, Zonis Zukerfeld, R. Adicción alimentaria: teoría, clínica y abordaje terapéutico. Universidad Austral; 2016.

3. Stoeckel LE, Weller RE, Cook EW 3rd, Twieg DB, Knowlton RC, Cox JE. Widespread reward-system activation in obese women in response to pictures of 
high-calorie foods.Neuroimage 2008; 41: 636-647.

4. Obregón A, Fuentes J, Pettinell P. Asociación entre adicción a la comida y estado nutricional en universitarios chilenos. Revista médica de Chile [Revista en línea] 2015 [acceso 9 Sept 2019 ]; 143(5). Disponible en: http://dx.doi.org/10.4067/S0034-98872015000500006

5. Meule, A. \& Gearhardt, A.N. Five years of the Yale Food Addiction Scale: Taking stock and moving forward Curr Addict Rep [Revista en línea] 2014 [acceso 9 Sept 2019] 1: 193. https://doi.org/10.1007/s40429-014-0021-z

6. Valdés M, Rodríguez M, Cervantes J, Camarena, B, de Gortar. P. Traducción al español de la escala de adicción a los alimentos de Yale (Yale Food Addiction Scale) y su evaluación en una muestra de población mexicana-Análisis factorial. Salud Mental 2016; 39(6): 295-302. Disponible en: http://www.medigraphic.com/pdfs/salmen/sam-2016/sam166c.pdf

7. Fondo Nacional de Recursos [En línea]. Montevideo. 2017. Programa de Prevención Secundaria Cardiovascular. [acceso 9 Sept 2019] Disponible en: http://www.fnr.gub.uy/descripcion_cardio

8. Organización de las Naciones Unidas para la alimentación y la agricultura [En línea]. The nutrition transition and obesity. [acceso 9 Sept 2019] Disponible en: http://www.fao.org/focus/S/obesity/obes2.htm

9. Zaragoza A, Ortiz R. Estado nutricional y nivel de actividad física según percepción de los estudiantes de la Universidad de Alicante. Rev Esp Nutr Comunitaria [Revista en línea] 2012 [acceso 9 Sept 2019] 18 (3): 149-153. Disponible en: https://fcsalud.ua.es/es/alinua/documentos/investigacion/articulo-nutricion-y-activ ida-fisica.pdf

10. Squiers L, Renaud J, Mc Comack L, Tzenq J, Bann C, Williams P. How accurate are Americans' perceptions of their own weight? J Health Commun [Revista en línea] 2014 [acceso 9 Sept 2019] (19)7: 795-812. Disponible en: https://www.ncbi.nlm.nih.gov/pubmed/24580374

11. Quintero J, Félix Alcántara M, Banzo-Arguis C, Martínez de Velasco Soriano R, Barbudo E, Silveria B, Pérez-Templado Ladrón de Guevara. Psicopatología en el paciente con obesidad. Salud Mental [revista en internet] 2016 [acceso 9 Sept 
12. Agüera Z, Wolz I, Sánchez I, Sauvaget A, Hilker I, Granero R, Jiménez-Murcia S, Fernández-Aranda F. Adicción a la comida un constructo controvertido. C. Med. Psicosom [Revista en línea] 2016 [acceso 9 Sept 2019] 117. Disponible en: http://www.editorialmedica.com/download.php?idart=727

13. Agüera Z, Wolz1 I, Sánchez I, Sauvaget A, Hilker I, Granero R, Jiménez-Murcia S, Fernández-Aranda F. Adicción a la comida un constructo controvertido. Revista Iberoamericana de Psicosomática [revista en internet]. No $117-2016$. Disponible en: http://www.editorialmedica.com/download.php?idart=727 


\section{ANEXO 1: FORMULARIO ADAPTADO YALE FOOD SCALE}

\&

FONDO NACIONAL

DE RECURSOS

Medicina Altamente Especializada
Fecha:

PACNUM:

Nombre:

Edad:

La siguiente encuesta pregunta aspectos de sus hábitos alimentarios en el último año, con el fin de contribuir a un estudio de prevalencia que será desarrollado por el equipo de nutrición de la institución.

Las preguntas apuntan a algunos alimentos que muchas veces se presenta dificultad de control ante su consumo, como por ejemplo alimentos dulces como el helado, el chocolate, las galletitas, tortas dulces, caramelos, otro tipo de alimentos ricos en harinas como las galletas, panes, pastas y arroz. También aquellos alimentos como papas fritas, hamburguesas, pizza y bebidas azucaradas.

Usted deberá responder a las siguientes afirmaciones con que frecuencia le sucede,

*Nunca (0)

*1 vez en el mes (1)

*2-4 veces en el mes (2)

*2-3 veces a la semana (3)

*4 o más veces a la semana (4)

1- He notado que cuando empiezo a comer esos determinados alimentos, he terminado comiendo más de lo que tenía planeado.

2- He notado que cuando estoy comiendo esos determinados alimentos he seguido comiéndolos, aunque ya no tengo hambre.

3- He notado que como tanto que llego a sentirme mal físicamente, por ejemplo, con inflamación, dolor de estómago, náuseas, indigestión.

4- Me preocupa que no he podido evitar consumir esos determinados alimentos o no he podido reducir su consumo.

5- He pasado mucho tiempo sintiéndome lento, pesado o cansado por haber comido de más.

6- He notado que a lo largo del día ingiero rápidamente esos determinados alimentos.

7- He notado que trato de obtener esos determinados alimentos cuando no están disponibles. Ejemplo: he ido a la tienda a conseguirlos, aunque haya tenido otras opciones en casa. 
8- En algunas ocasiones cuando he consumido esos determinados alimentos de forma tan frecuente o en tan grandes cantidades, he dejado de trabajar, de convivir con la familia o de realizar otras actividades para disfrutarlo.

9- En algunas ocasionas cuando he consumido esos determinados alimentos de forma tan frecuente o en tan grandes cantidades, he pasado el tiempo envuelto en sentimientos negativos (culpa, baja autoestima) porque he comido de más, en lugar de trabajar, de convivir con la familia o de realizar otras actividades que disfruto.

10- He evitado asistir a eventos sociales o laborales al saber que esos determinados alimentos estarán disponibles, por miedo a comer de más.

11- He evitado asistir a eventos sociales o laborales porque no había esos determinados alimentos y no podía consumirlos.

12- Me he sentido inquieto, ansioso o agitado cuando he dejado de consumir esos determinados alimentos (no considerar a las bebidas con cafeína).

13- He consumido esos determinados alimentos para evitar sentimientos de ansiedad o de inquietud producidos por haber dejado de comerlos (no considerar a las bebidas con cafeína, como los energizantes, café, etc.).

14- He notado que tengo un gran deseo o urgencia por consumir esos determinados alimentos cuando dejo de comerlos.

15- Mi comportamiento con respecto a los alimentos y mi forma de comer me genera malestar (ansiedad, culpa, depresión).

16- Por culpa de la comida y la alimentación tengo dificultades importantes para desenvolverme de un modo adecuado en mis diferentes actividades (rutina diaria, trabajo, sociales, familiares, dificultades de salud).

En los últimos 12 meses: SI - NO

\begin{tabular}{|l|l|}
\hline $\begin{array}{l}\text { 17- Mi consumo de alimentos me ha causado sensaciones de depresión, } \\
\text { ansiedad o culpa. }\end{array}$ & \\
\hline $\begin{array}{l}\text { 18- Mi consumo de alimentos me ha causado importantes problemas físicos } \\
\text { (diabetes, intolerancia a la glucosa, hipertensión, obesidad) o ha } \\
\text { complicado los ya existentes. }\end{array}$ & \\
\hline $\begin{array}{l}\text { 19- He continuado comiendo el mismo tipo de alimentos en la misma } \\
\text { cantidad de alimentos a pesar de que me han ocasionado problemas } \\
\text { psicológicos o físicos (ansiedad, culpa, diabetes, intolerancia a la } \\
\text { glucosa, hipertensión, obesidad). }\end{array}$ & \\
\hline $\begin{array}{l}\text { 20- Conforme pasa el tiempo he notado que necesito comer más y más para } \\
\text { lograr el estado de bienestar que deseo, como por ejemplo para reducir } \\
\text { mis emociones negativas (ansiedad o culpa) e incrementar el placer. }\end{array}$ & \\
\hline $\begin{array}{l}\text { 21- He notado que comer la misma cantidad de comida ya no reduce mis } \\
\text { emociones negativas o que ya no aumentan las sensaciones placenteras } \\
\text { como antes lo hacía. }\end{array}$ & \\
\hline $\begin{array}{l}\text { 22- He hecho frenar o disminuir mi consumo de esos determinados } \\
\text { alimentos. }\end{array}$ & \\
\hline $\begin{array}{l}\text { 23- He hecho algo por frenar o disminuir mi consumo de esos determinados } \\
\text { alimentos. }\end{array}$ & \\
\hline
\end{tabular}


24- He tenido éxito al frenar o disminuir mi consumo de esos determinados alimentos.

25- ¿Cuántas veces en el último año he intentado frenar o disminuir el consumo de esos determinados alimentos?

\begin{tabular}{|l|l|}
\hline$* 1$ o menos veces & \\
\hline *2 veces & \\
\hline$* 3$ veces & \\
\hline *4 veces & \\
\hline *5 o más veces & \\
\hline
\end{tabular}

